\title{
Tratamiento del hombro doloroso; ¿antiinflamatorio no esteroideo (AINE) o infiltración?
}

\author{
V. Chavero Carrasco, O. Salguero M olpeceres, \\ P. López Ruano, S. Álvarez M ONTERO* \\ Médicos Residentes de $3^{\text {e }}$ año M edicina de Familia y Comunitaria. Centro de Salud \\ Universitario Pozuelo I. Madrid. *M édico de Familia. Tutor M IR \\ de M FyC. Centro de Salud Torrelodones. Madrid
}

\section{RESUMEN}

En un ensayo clínico aleatorizado, doble ciego sobre 100 pacientes vistos en una consulta de Reumatología se observó que, tras un seguimiento de 4 semanas, el tratamiento del hombro doloroso con triamcinolona infiltrada fue superior al naproxeno en cuanto a mejoría del dolor $(p=0,04)$, pero no en la abducción activa y la limitación funcional (no hubo diferencias estadisticamente significativas). Tampoco hubo diferencias significativas en cuanto a las remisiones.

Palabras clave: Hombro. AINEs. Infiltración in traarticular. Dolor de hombro. Tratamiento.
Treatment of shoulder pain; non steroidal anti-inflam matory drug (NSAID) or intraarticular inyection?

\section{ABSTRACT}

In a double blinded randomized clinical trial on 100 patients seen in a Rheumatologic clinic was observed that, after a follow up of 4 weeks, the treatment of the painful shoulder with triamcinolo na injections was superior to naproxeno for impro vement of the pain $(p=0.04)$, but not in the active abduction and the functional limitation (there were not differences statistically significant). Neither there were significant differences for the remis sions.

Key words: Shoulder. NSAIDs. Intraarticular in yection. Shoulder pain. Treatment.

\section{ESCENARIO CLÍNICO}

El hombro doloroso es una patología muy frecuente en Atención Primaria. Las opciones de tratamiento son múltiples y se hace necesario definir la efectividad de las mismas para ofrecer la opción terapéutica más eficaz. Se planteó la duda sobre la eficacia del uso de AINE frente a la inyección local de corticoides.

\section{PREGUNTA ESTRUCTURADA}

¿En el hombro doloroso (tema) qué opción es más eficaz, AINEs o infiltración (intervención), para reducir el dolor y la impotencia funcional (resultado)?

\section{BÚSQUEDA}

\section{Términos de búsqueda}

"(Shoulder AND nsaid and intraarticular injec tion) OR (shoulder pain AND treatment)" (hombro y AINE y inyección intraarticular o dolor de hombro y tratamiento). La búsqueda se hizo a través de OVID, seleccionando las bases de datos: EBM reviews (Cochrane Database of Systematic Reviews

Aceptación: 04-03-02 
2 Quarter 2001), EBM reviews (Cochrane controlled Trails Register 3 Quarter 2001) EBM ACP (Journal Club 1991 a marzo /abril 2001), MEDLINE (1966 a la $1^{\text {a }}$ semana de Octubre 2001), EMBASE (1980 a 2001 semana 40 y TRYP data base, en Octubre de 2001.

Las mejores evidencias se logran con un buen metaanálisis o con una buena revisión sistemática. La realización de los mismos requiere un gran esfuerzo por lo que se aleja de nuestros objetivos y posibilidades. La revisión sistemática y el metaanálisis deberían analizar absolutamente todos los estudios al respecto, realizando una selección de los más válidos en cuanto a la metodología seguida. Con la consideración en conjunto de un mínimo de estudios suficientemente válidos se obtienen conclusiones. Cuando los estudios no son suficientes en cuanto a calidad, número de pacientes, homogeneidad, etc., la conclusión de la revisión sistemática es que no existe suficiente evidencia.

En la búsqueda realizada se encontraron dos revisiones sistemáticas, con fechas de $1995^{1}$ y $1993^{2}$ (la revisión realizada por Green et al. ${ }^{1}$ se encuentra actualizada hasta 1999 en la librería Cochrane) ambas concluyeron que no era suficiente la calidad, número y homogeneidad de los estudios.

Pero esta conclusión de una revisión sistemática no implica que el clínico deba necesariamente inhibirse, pues la realidad demanda soluciones en beneficio de los pacientes. Para tomar decisiones, a menudo al clínico no le queda más remedio que aplicar de forma explícita y juiciosa (contando con valores y circunstancias) sólo la mejor evidencia existente.

La pregunta es respecto a una intervención, la mayor evidencia, después del metaanálisis y la revisión sistemática, son los ensayos clínicos aislados. El ensayo clínico seleccionado se obtuvo por una doble vía: a través de una búsqueda en Medline, y por resultar destacado como ensayo válido en las revisiones sistemáticas publicadas hasta el momento de la búsqueda.

En concreto se encontraron tres estudios con la suficiente calidad: el primero de ellos; Adebajo (1990) sólo comparaba AINEs con placebo, el segundo; Berry (1980) comparaba AINEs sólo con infiltración más AINEs por lo que se desestimaron los dos. El estudio seleccionado fue realizado por Petri et $\mathrm{al}^{3}$.

—Diseño: ensayo clínico aleatorizado, doble ciego.

-Población: se seleccionaron 100 pacientes; 69 hombres y 31 mujeres, vistos en una consulta de Reumatología.

- Criterios de inclusión: debían cumplir al menos dos de los siguientes: dolor a la abducción a cualquier grado de movimiento; dolor al movimiento entre $45^{\circ}$ y $120^{\circ}$, y/o sensibilidad a la presión en la inserción de tendón del supraespinoso.
-Criterios de exclusión: artritis de hombro, infiltración del supraespinoso en los tres meses previos, sospecha de rotura del tendón, contraindicaciones del uso de AINEs y alergia a la lidocaína.

-Intervención: aleatoriamente se distribuyeron en cuatro grupos de 25 pacientes con estos tratamientos:

- Una inyección subacromial de 4cc de lidocaína más naproxeno $500 \mathrm{mg} / 12$ horas vía oral (v.o.).

- Una inyección subacromial de 3 cc de lidocaína-1cc de triamcinolona más v.o. naproxeno 500 $\mathrm{mg} / 12$ horas.

- Una inyección subacromial de 3 cc de lidocaína-1 cc de triamcinolona más v.o. placebo cada 12 horas.

- Inyección subacromial de 4 cc de lidocaína más v.o. placebo cada 12 horas. El tratamiento oral se mantuvo cuatro semanas y se valoró el resultado a las dos y a las cuatro semanas.

-Variables de efecto: se comparó la abducción activa medida en grados, el dolor y la limitación de función con una escala cualitativa.

—Resultados: se observó que la infiltración con triamcinolona produjo una mejoría estadísticamente significativa frente a lidocaína sobre las tres variables; grado de abducción activa, limitación de función y dolor, tanto en la segunda como en la cuarta semana. El tratamiento con triamcinolona fue superior al naproxeno, en cuanto a mejoría de dolor $(\mathrm{p}=$ $0,04)$ y en el indice clínico $(\mathrm{p}=0,04)$ pero no en la abducción activa y limitación funcional.

A las dos semanas el tratamiento con triamcinolona mostraba efectos significativos sobre las cuatro variables. El tratamiento con naproxeno también mejoraba esas variables pero las diferencias no eran estadísticamente significativas.

A las cuatro semanas, el tratamiento con triamcinolona continuaba siendo superior al del placebo en términos de abducción activa, dolor e índice clínico. El tratamiento con naproxeno no mostraba mejorías estadísticamente significativas frente a las del placebo a las cuatro semanas.

En el porcentaje de mejoría que se observaba en cada tratamiento el naproxeno promediaba una influencia del $2,6 \%$ con $\mathrm{p}=0,02$ y triamcinolona una influencia del $13,1 \%$ con $\mathrm{p}=0,0001$. Calculado el NNT para la variable remisión los resultados no mostraban diferencias estadísticamente significativas entre los dos tipos de tratamiento y entre éstos y el del placebo. La combinación de triamcinolona y naproxeno no tuvo variaciones estadísticamente significativas lo que sugiere que no presentan efectos sinérgicos.

\section{COMENTARIOS}

Las intervenciones que han demostrado utilidad en el tratamiento del hombro doloroso son la infiltración con corticoides más distensión de la cápsu- 
la articular con $19 \mathrm{ml}$ de lidocaína al 0,5\% de forma repetida y con control ecográfico (en un pequeño ensayo clínico) y la destrucción de los depósitos de calcio con ondas de choque en el caso de la tendinitis con calcificaciones (un pequeño ensayo clínico). Aún no hay evidencias claras sobre la eficacia de analgésicos como el paracetamol, los AINEs, orales, en forma de cremas o infiltrados, la inyección de corticoides en regimen subacromial o intra-articular, la fisioterapia, el láser, la electroterapia, el frío, la guanetidina intra-articular, la cirugía o la estimulación nerviosa cutánea ${ }^{4}$.

Esto es debido a que existe una falta de homogeneidad en el concepto de hombro doloroso (abarca varios problemas muy diferentes), las poblaciones estudiadas, las intervenciones realizadas y en las medidas de resultados empleadas para valorar la eficacia.

\section{Validez}

Siguiendo las pautas de Guyatt et al. ${ }^{5}$ el artículo responde a una pregunta claramente definida, muy similar a la que nosotros nos planteábamos, los pacientes son distribuidos de una manera aleatoria entre los distintos grupos. Se informa que el seguimiento fue mayor del $80 \%$, pues el $98 \%$ de los pacientes fue analizado a las dos semanas y el $95 \%$ se analizó a las cuatro semanas. Y el estudio fue realizado a doble ciego, siendo tratados los pacientes de la misma forma.

Como puntos débiles hay que resaltar: que no podemos saber si esta secuencia de aleatorización se realizó de manera oculta, que se trata de una muestra pequeña, con pacientes seleccionados fuera del ámbito de Atención Primaria, y que no se especifica si los grupos fueron homogéneos.

\section{Importancia}

Es difícil evaluar el impacto de la infiltración frente a AINEs dado que se han medido los resulta- do con escalas clínicas. El NNT sólo se pudo calcular para la variable remisión completa ya que era la única dicotómica y en este caso las diferencias no son estadísticamente significativas. En cualquier caso parece que las diferencias entre una otra opción no son.

\section{Aplicabilidad}

Los tratamientos para el hombro doloroso más habitualmente al alcance de los médicos de familia son paracetamol, AINEs (orales o tópicos), infiltraciones y fisioterapia. Ninguno con una eficacia claramente demostrada.

A los pacientes con un hombro doloroso de características similares a los pacientes de este estudio (con al menos dos de estas tres características: dolor a la abducción a cualquier grado de movimiento; dolor al movimiento entre 45 y 120 grados y sensibilidad a la presión en la inserción del supraespinoso) en los que se plantee optar entre AINEs orales o una infiltración, habrá que informarles que las infiltraciones parecen ligeramente superiores a los AINEs orales, en qué consisten ambos tratamientos y en los posibles efectos secundarios de los mismos.

Sería conveniente contar con buenos estudios realizados en el ámbito de Atención Primaria. Mientras tanto, habrá que seguir tomando decisiones de forma juiciosa, contando con las escasas evidencias disponibles.

CORRESPONDENCIA:

Victor Chavero Carrasco

C/ Benito Gutierrez, 11 S-2

28008 Madrid

e-mail: victor_chavero_carrasco@hotmail.com

\section{Bibliografía}

1. Green S, Buchbinder R, Gloazier R, Forbes A. Systematic review of randomised controlled trials of interventions for painful shoulder: selection criteria, outcome assessment and efficacy. BMJ 1998; 316: 354-360. Search date 1995; primary sources Medline and hand searched references.

2. Van Der Wintd D, Van Der Heiden GJMG, Scholten RJPM, Koes BW, Bouter LM. The efficacy of non-steroidal antinflammatory drugs (NSAIDs) for shoulder complaints. A systematic review. J Clin Epidemiol 1995; 48: 691-704. Search date 1993; primary sources Medline and hand sear- ched references.

3. Petri M, Dobrow R, Neeima R, Whinting-O'Keefe Q. Randomized, double-blind, placebo-controlled estudy of the treatment of the painful shoulder. Artritis\&rheumatism 1987; 30: 1040-5.

4. Speed C, Hazelman B. Shoulder pain. En: Clinical Evidence 2001; 5. p. 850-64.

5. Guyatt GH, Sackett DL, Cook DJ. Users'guides to the medical literature, II. How to use an article about terapy or prevention. JAMA 1993; 270: 2598-601 \& JAMA 1994; 271: 59-63. 